\title{
The Noradrenergic Neurotoxin DSP-4 Eliminates the Coeruleospinal Projection but Spares Projections of the A5 and A7 Groups to the Ventral Horn of the Rat Spinal Cord
}

\author{
W. Ernest Lyons, Jean-Marc Fritschy, and Reinhard Grzanna \\ Department of Neuroscience, Johns Hopkins University School of Medicine, Baltimore, Maryland 21205
}

\begin{abstract}
Systemic administration of the noradrenergic neurotoxin DSP-4 results in a complete loss of staining of noradrenergic (NA) axons in the dorsal horn and intermediate zone of the rat spinal cord. NA axon staining in the ventral horn and in the intermediolateral cell column is only slightly decreased by the drug treatment. We have taken advantage of this differential effect of DSP-4 on NA axons to determine the location and number of cells that give rise to NA axons in the ventral horn and the intermediolateral cell column. Retrograde transport of the fluorescent tracer True blue was combined with dopamine- $\beta$-hydroxylase immunohistochemistry 2 weeks after treatment of rats with $50 \mathrm{mg} / \mathrm{kg}$ of DSP. 4. Compared with controls, the drug treatment resulted in a more than $90 \%$ decrease in the number of retrogradely labeled NA neurons in the locus coeruleus and an only 30$50 \%$ reduction in the number of retrogradely labeled NA cells in the $A 5$ and $A 7$ groups. The results reveal different sites of termination in the spinal cord of NA axons originating in the LC and in NA cells of the A5 and A7 groups: the LC distributes fibers mainly to the dorsal horn and the intermediate zone, while NA cells of the A5 and A7 groups project to motoneurons of the ventral horn and the intermediolateral cell column.
\end{abstract}

Descending noradrenergic (NA) axons are distributed to all levels of the rat spinal cord. The highest density of NA axon terminals is found in the vicinity of preganglionic sympathetic neurons, among somatic motoneurons, and in the most superficial part of the dorsal horn (Carlsson et al., 1964; Dahlstrom and Fuxe, 1965; Mizukawa, 1980; Westlund et al., 1983). Retrograde transport studies have identified 4 distinct NA subgroups as the source of noradrenergic afferents to the rat spinal cord: the locus coeruleus (LC), the subcoeruleus, and the A5 and A7 groups (Satoh et al., 1977; Blessing et al., 1981; Westlund et al., 1983; Byrum et al., 1984; Loewy et al., 1986). In their original description of the bulbospinal monoamine systems, Dahlstrom and Fuxe (1965) presented evidence for the existence of 2 distinct descending bulbospinal NA fiber systems with different sites of termination: a large fiber system coursing through the anterior funiculus and the most ventral part of the lateral funic-

\footnotetext{
Received June 6, 1988; revised Sept. 14, 1988; accepted Sept. 20, 1988

Wc wish to thank Dr. S. B. Ross, $\Lambda$ stra Lakemedel $\Lambda \mathrm{B}$, Sweden, for a generous gift of DSP-4. This work was supported by USPHS Grant NIMH MH-41977.

Correspondence should be addressed to Reinhard Grzanna, Ph.D., Johns Hopkins University School of Medicine, Department of Neuroscience, $725 \mathrm{~N}$. Wolfe Street, Baltimore, MD 21205

Copyright $\mathrm{C} 1989$ Society for Neuroscience $0270-6474 / 89 / 051481-09 \$ 02.00 / 0$
}

ulus innervating the ventral horn, and a second pathway running in the latcral funiculus, especially in its dorsal part, innervating the dorsal horn and the intermediolateral cell column.

Although there is a general consensus that different NA cell groups are likely to innervate different regions within the spinal cord, details of the distribution of axons from each NA subgroup within the cord are not yet known. Most authors agree that the LC is the principal source of the NA input to the ventral horn and to much of the intermediate zone of the spinal cord. This conclusion has been derived from autoradiographic anterograde tracing studies and from studies of the effects of bilateral LC lesions on the distribution of NA axons in the spinal cord ( $\mathrm{Ny}-$ gren and Olson, 1977; Commissiong et al., 1978; Holstege et al., 1979; Commissiong, 1981; Kuypers, 1982; Holstege and Kuypers, 1987). That the LC may not distribute axons to lamina IX of the ventral horn was first suggested by Martin et al. (1979a, b), who traced descending projections from the LC to the spinal cord of the opossum. The first direct demonstration of LC projections to the dorsal horn and the lack of LC projections to lamina IX of the ventral horn of the rat spinal cord was reported by Fritschy et al. (1987). These authors combined anterograde transport of the lectin Phaseolus vulgaris leucoagglutinin (PHAL) with dopamine- $\beta$-hydroxylase $(\mathrm{DBH})$ immunohistochemistry and demonstrated unequivocally a preferential distribution of NA axons of the LC to the dorsal horn and the intermediate zone but found only a sparse distribution of LC axons in the ventral horn. Less controversial is the issue of the source of the dense NA input to the intermediolateral cell column of the thoracic spinal cord. It is now widely accepted that cells of the A5 group are the principal source of the NA input to the intermediolateral cell column (Byrum et al., 1984; Loewy et al., 1986), although it is still not known to what extent other NA subgroups may contribute to this dense terminal plexus at thoracic spinal cord levels. Almost nothing is known about the termination of NA axons originating in the A7 group, even though this NA subgroup emits extensive projections to the spinal cord (Stevens et al., 1982; Westlund et al., 1983).

Methodological difficulties have hampered definitive identification of the sites of termination of axons from different NA subgroups within the spinal cord. The A5 and A7 groups do not form compact clusters of cells and hence are not readily lesioned to reveal the distribution of their axons at spinal cord levels. For the same reasons, anterograde tracing experiments are of only limited value. Retrograde tracing studies are also not optimally suited to uncover differential NA projections to the spinal cord since they would require very small and discrete injections of tracer into different regions of the gray matter. An 
elegant approach for the study of the NA bulbospinal projection was employed by McBride et al. (1985). These investigators analyzed the retrograde labeling of NA cells from the spinal cord after neonatal treatment of rats with the catecholamine neurotoxin 6-hydroxydopamine and discovered that this drug treatment results in degeneration of coeruleospinal axons.

In a previous study, we characterized the effects of the neurotoxin DSP-4 ( $N$-(2-chloroethyl)- $N$-ethyl-2-bromobenzylamine; Ross et al., 1973; Zieher and Jaim-Etcheverry, 1980; Jonsson et al., 1981) on the distribution of NA axons in the rat CNS. In the spinal cord, systemic administration of this drug produces a complete loss of NA axon staining in the dorsal horn and intermediate zone but has little effect on NA axon terminals innervating the intermediolateral cell column and the ventral horn (Fritschy and Grzanna, 1989). In this study, we have taken advantage of this highly selective neurodegenerative effect of DSP-4 on NA axons to identify the cells that give rise to the NA axon terminals in the intermediolateral cell column and the ventral horn of the spinal cord. Using retrograde transport of the fluorescent tracer True blue (TB) in combination with DBH immunohistochemistry after DSP-4 treatment, we demonstrate that NA axons innervating somatic and visceromotor neurons originate in cells of the A5 and A 7 groups.

\section{Materials and Methods}

Neurotoxin injections. Adult male Sprague-Dawley rats weighing 150 $175 \mathrm{gm}$ were used in all experiments. Rats received a single dose of 50 $\mathrm{mg} / \mathrm{kg}$ of freshly prepared DSP-4, administered i.p. in sterile saline. Previous studies have shown that this dose produces degeneration of LC axons in forebrain regions and in the spinal cord (see Jonsson, 1983). Because of the very short half-life of DSP-4 in solution (Ross et al., 1973), the drug was dissolved immediately prior to injecting each animal.

NA axon staining in spinal cord. For analysis of NA axon distribution in the spinal cord of untreated and DSP-4 treated rats, sections were processed for immunohistochemistry using antibodies to rat $\mathrm{DBH}$ (Grzanna and Coyle, 1976) in combination with the avidin-biotin method of Hsu et al. (1981). Rats were anesthetized with chloral hydrate and perfused through the ascending aorta using a modification of the $\mathrm{pH}$ shift method of Berod et al. (1981). Perfusions were started with 100 $\mathrm{ml}$ of ice-cold PBS followed by $400 \mathrm{ml}$ of $100 \mathrm{~mm}$ sodium acetate buffer, pII 6.5 , containing $4 \%$ paraformaldehyde and then $400 \mathrm{ml}$ of $100 \mathrm{~mm}$ borate buffer, $\mathrm{pH} 9.5$, containing $4 \%$ paraformaldehyde and $0.1 \%$ glutaraldehyde. Brains and spinal cords were promptly removed, postfixed for 4-6 $\mathrm{hr}$ and then transferred into PBS containing $10 \%$ dimethylsulfoxide for cyoprotection. Thirty micron transverse sections through the brain stem and spinal cord were incubated free-floating in a 1:2000 dilution of guinea pig anti-rat DBH in PBS containing $0.2 \%$ Triton $X-100$ and $1 \%$ normal goat serum. After 3 washes in PBS, sections were incubated for $1 \mathrm{hr}$ at room temperature with biotinylated goat antiguinea pig immunoglobulins, washed again, and then incubated with the avidin-biotin-peroxidase complex for $1 \mathrm{hr}$. To visualize the antigen, sections were reacted in a cocktail of $0.05 \%$ diaminobenzidine and $0.005 \%$ hydrogen peroxide in $50 \mathrm{~mm}$ Tris buffer at $\mathrm{pH} 7.6$

For intensification of staining, sections were further processed according to the procedure described by Gerfen (1985).

Retrograde transport experiments. The fluorescent retrograde tracer TB was injected into either thoracic ( 4 control rats and 4 DSP-4-treated rats) or lumbar spinal cord levels (2 control rats and 2 DSP-4-treated rats). DSP-4 treated rats were injected with TB $14 \mathrm{~d}$ after drug treatment. For tracer injections, rats were anesthetized with chloral hydrate and segments of either thoracic or lumbar spinal cord were exposed by laminectomy of 2 adjacent vertebrae. TB was injected via a glass micropipet (tip diameter, $75-100 \mu \mathrm{m}$ ) mounted onto a $10 \mu$ l Hamilton syringe. Five $100 \mathrm{nl}$ injections of a $2.5 \%$ sonicated suspension of TB were made at approximately $300 \mu \mathrm{m}$ intervals. No attempt was made to restrict the tracer to one side of the spinal cord.

Immunohistochemistry after retrograde transport. Fourteen days after delivery of the retrograde tracer, rats were perfused as described above for staining of NA axons in spinal cord sections. Following overnight incubation of sections in the primary antibody, sections were washed with PBS and then incubated for $1 \mathrm{hr}$ in a 1:50 dilution of fluorescein isothiocyanate (FITC) conjugated goat anti-guinea pig antiserum. All sections were mounted onto gelatin-chrome-alum-coated glass slides and air-dried.

Data collection. Fluorescent-labeled cells were viewed in a Leitz Ortholux fluorescence microscope equipped with a Ploem incident illuminator. TB-labeled and DBH-positive cells were identified in the same tissue section using different filter combinations. For collection of data, NA cells of the pons were delineated into subgroups according to the nomenclature of Dahlstrom and Fuxe (1964); rostral and caudal A5 groups were distinguished based upon the criteria reported previously (Grzanna et al., 1987). In each section through the pons, retrogradely labeled DBH-positive cells were counted in the A5 and A7 groups. The number of retrogradely labeled neurons in the LC was determined using photographs of the LC that were taken from every section containing this nucleus. For the description of NA axons in the spinal cord, the atlas by Paxinos and Watson (1986) was consulted. Fluorescence photomicrographs and dark-field photomicrographs of NA axons stained with the avidin-biotin procedure were taken using Kodak Tmax 400 film.

\section{Results}

\section{Effects of DSP-4 on NA axons in spinal cord}

In spinal cord sections from control rats, DBH immunohistochemical staining reveals an extensive network of NA axons throughout the gray matter at all spinal cord levels (Figs. 1A, $2 A$ ). In the ventral horn a dense plexus of NA axon terminals is present in the vicinity of motoneurons. The highest density of NA axon terminals is found in the intermediolateral cell column of the thoracic spinal cord (Fig. $2 A$ ). Fourteen days after systemic injections of $50 \mathrm{mg} / \mathrm{kg}$ of DSP-4, DBH immunohistochemistry reveals that all NA axon staining is abolished in the dorsal horn and the intermediate zone (Figs. $1 B$ and $2 B$ ). Extensive loss of NA axon staining is also observed in lamina VII of the ventral horn and in the lamina IX surrounding the central canal. In contrast, the density of NA axon terminals in lamina IX of the ventral horn is only slightly reduced. This plexus is present in both the lateral and medial motoneuron pools. Immunohistochemical staining of the very dense NA axon terminal plexus in the intermediolateral cell column of the thoracic spinal cord is also not affected by DSP-4 treatment (Fig. 2B).

The extent of the loss of NA axon staining in the ventral horn varies from animal to animal, but the staining of NA axons in motoneuron pools at $14 \mathrm{~d}$ is generally not different from that seen at $28 \mathrm{~d}$ after drug treatment. Preliminary studies of the long-term effects of DSP-4 suggest that 3 months after drug treatment the staining of these remaining NA axons in the ventral horn and the intermediolateral cell column is less intense than after 2 weeks. Thus, loss of NA axons staining is most dramatic during the first 2 weeks after drug treatment; the additional loss of axon staining after the second week is much less conspicuous and appears to occur at a much slower rate.

\section{Retrograde tracer experiments after DSP-4 treatment}

Fourteen days after injections of TB into thoracic and lumbar segments of the spinal cord of untreated rats, retrogradely labeled NA cells are found in the LC, subcoeruleus, and the A5 and $A 7$ groups (Table 1; Figs. 3, 4). For the retrograde transport studies in DSP-4-treated rats, we have injected TB $14 \mathrm{~d}$ after drug treatment, since the loss of NA axon staining is nearly complete by the end of the second week. As in untreated rats, these injections labeled non-NA cells throughout the pontine 

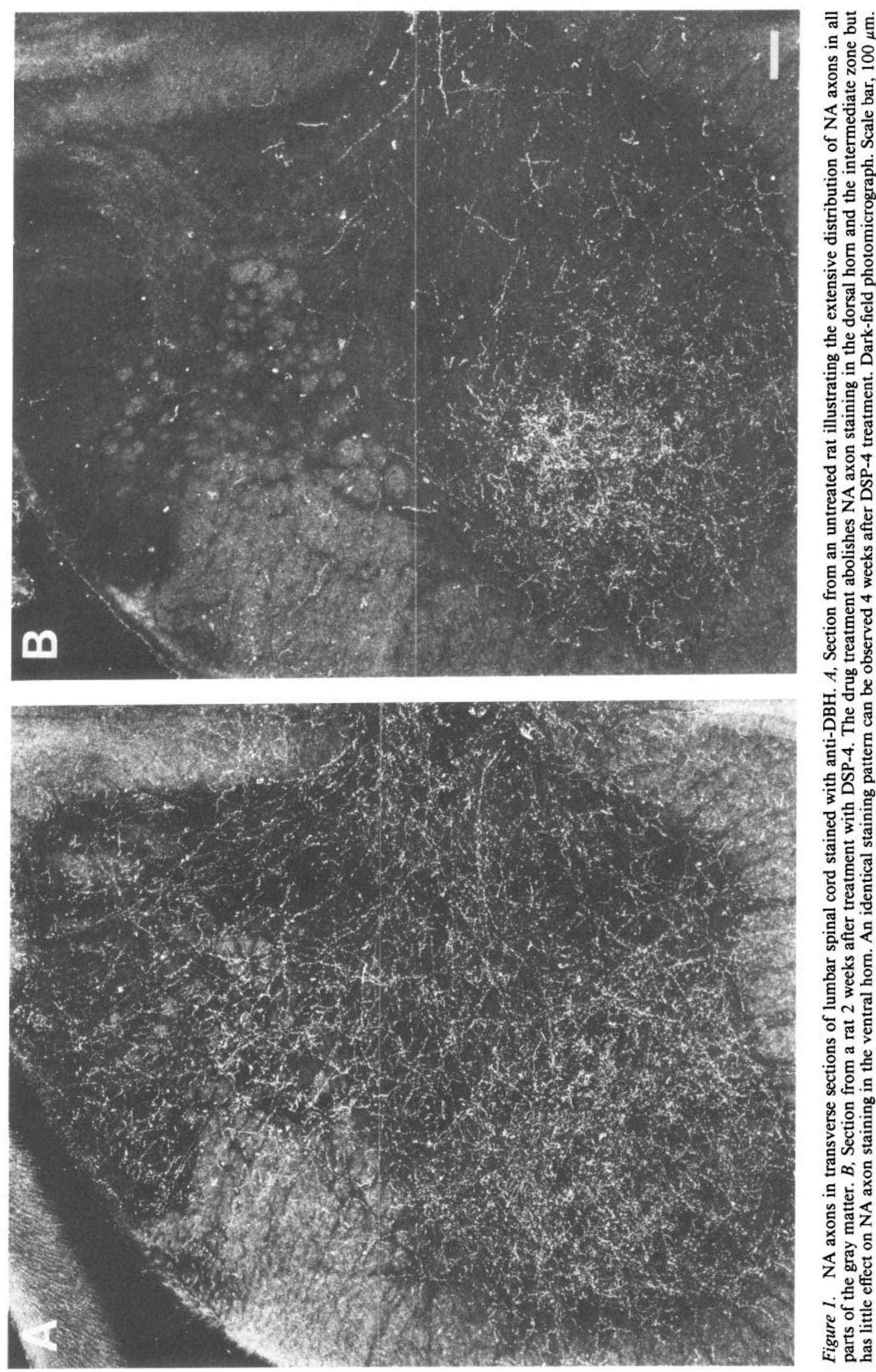

동

奥卷

苞苞

जิ

青少

运息

焉节

它究部

늠

急定

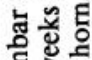

罗需

행ㄷㅇ

范

틍

듬ำ

证

可

평

言艺

ㄷ․

胥。

Z

它政

今

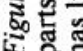



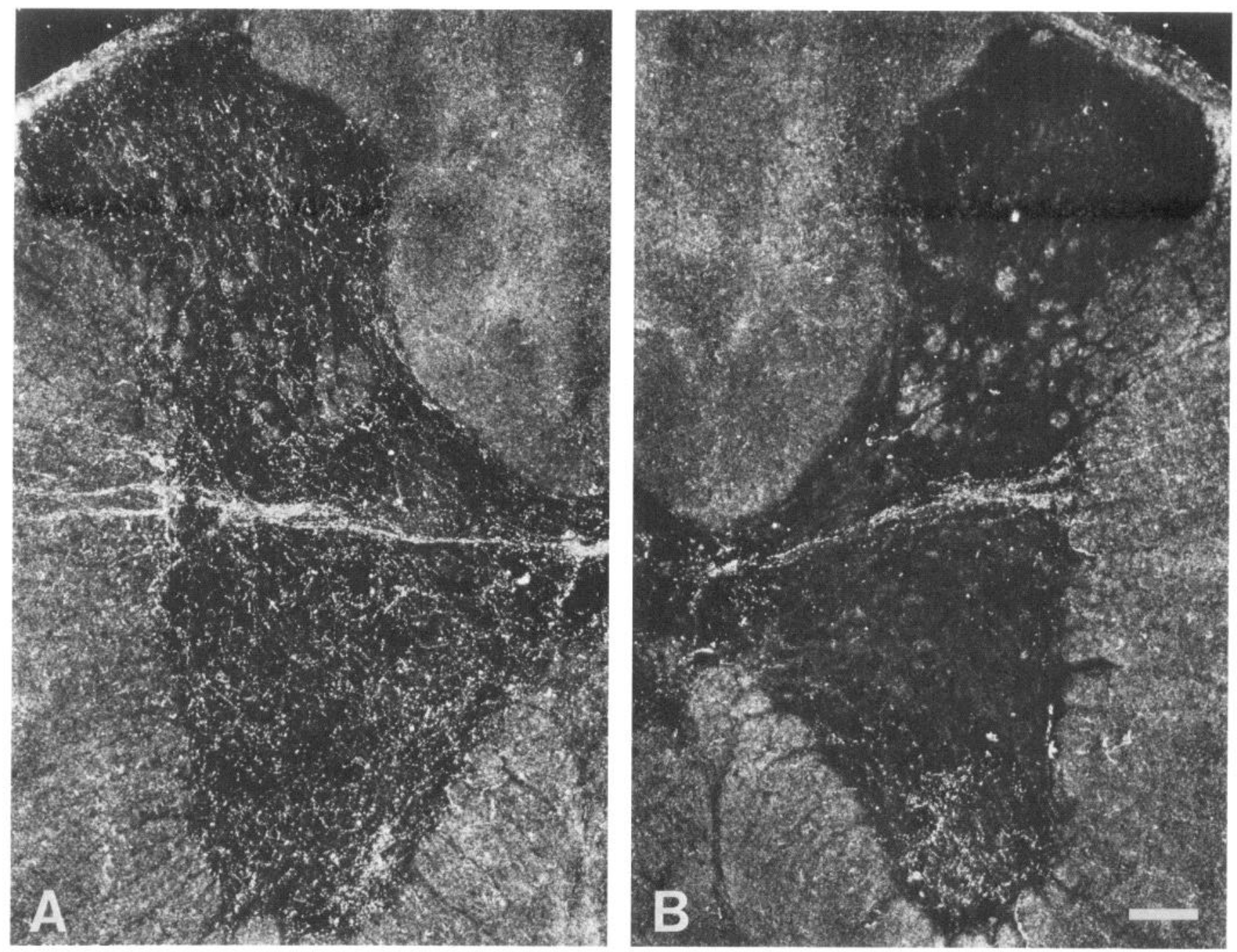

Figure 2. NA axons in transverse sections of thoracic spinal cord. A, Section from a control animal. The highest density of NA axon terminals is present in the intermediolateral cell column. $B$, Section from a rat 2 weeks after treatment with DSP-4. There is complete loss of NA axon staining throughout the gray matter with sparing of NA axons in the intermediolateral cell column and the ventral horn. Dark-field photomicrograph. Scale bar, $100 \mu \mathrm{m}$.

reticular formation with an intensity not different from that seen in control rats. The intensity of immunohistochemical staining of NA cell bodies in DSP-4-treated rats was also not different from the staining seen in sections from untreated animals.

Injections of TB into thoracic levels of the spinal cord 2 weeks after DSP-4 treatment labeled very few cells in the LC (Fig. 3). In control animals, these injections labeled on average 572 cells in the LC bilaterally. In DSP-4-treated rats, only 39 retrogradely labeled cells were counted, a reduction of $93 \%$. In the subcoeruleus, 123 cells were counted in control rats and 48 were found in DSP-4-treated rats. NA cells in the dorsal part of the subcoeruleus, which lies immediately ventral to the LC outside the central gray, were not retrogradely labeled, while there was substantial retrograde labeling of NA cells in the more ventral part of the subcoeruleus, the portion that extends rostrally and laterally to the A7 group and ventrally toward the rostral A5 group.

Injections of TB into thoracic segments of the cord resulted in substantial labeling of NA cells in the A5 and A7 groups (Fig. 4). In control rats, these injections labeled on average 120 cells in the caudal A5 group, 142 cells in the rostral A5 group, and
178 cells in the A7 group. In DSP-4-treated rats, injections of TB labeled 62 cells in the caudal A5 group ( $52 \%$ of control), 46 cells in the rostral A5 group (32\% of control), and 90 cells in the A7 group ( $51 \%$ of control).

To determine whether a different pattern of retrograde labeling can be observed after tracer injections into lumbar segments of the spinal cord, TB was deposited at low lumbar levels of 2 control rats and 2 DSP-4-treated rats. These injections labeled bilaterally 473 cells in the LC of control rats and 37 cells in DSP-4-treated rats (8\% of control). In the subcoeruleus, 109 cells were labeled bilaterally in untreated rats and 51 cells in drug-treated animals ( $47 \%$ of control). Of the NA cells retrogradely labeled, none were found in the cluster of cells located immediately ventral to the LC just outside the central gray. Substantial labeling was observed in the A5 and A7 groups bilaterally: 136 cells in the caudal A5 group, 120 cells in the rostral A5 group, and 186 cells in the A7 group. In DSP-4treated rats, lumbar injection of TB-labeled 86 cells in the caudal A5 group (65\% of control), 68 cells in the rostral A5 group (57\% of control), and 121 in the $\mathrm{A} 7$ group (63\% of control). Table 1 

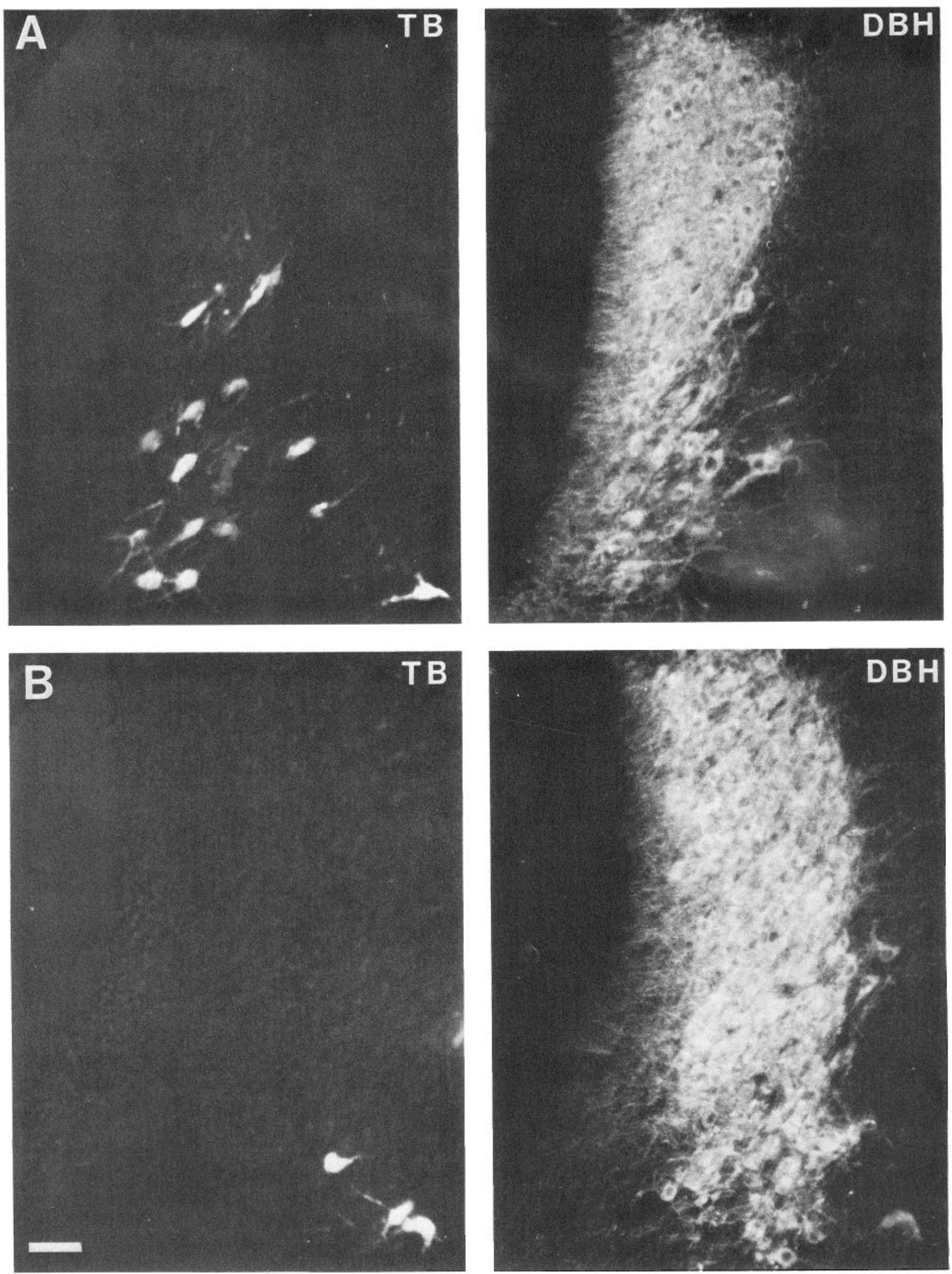

Figure 3. Pair of fluorescence photomicrographs of retrograde labeling of LC after TB injections into thoracic segments of the spinal cord. $A$, Retrograde labeling of cells in the caudal LC of control rat. $B$, Section through the caudal LC of a rat treated with DSP-4 2 weeks before tracer injections into the spinal cord. Scale bar, $50 \mu \mathrm{m}$. 

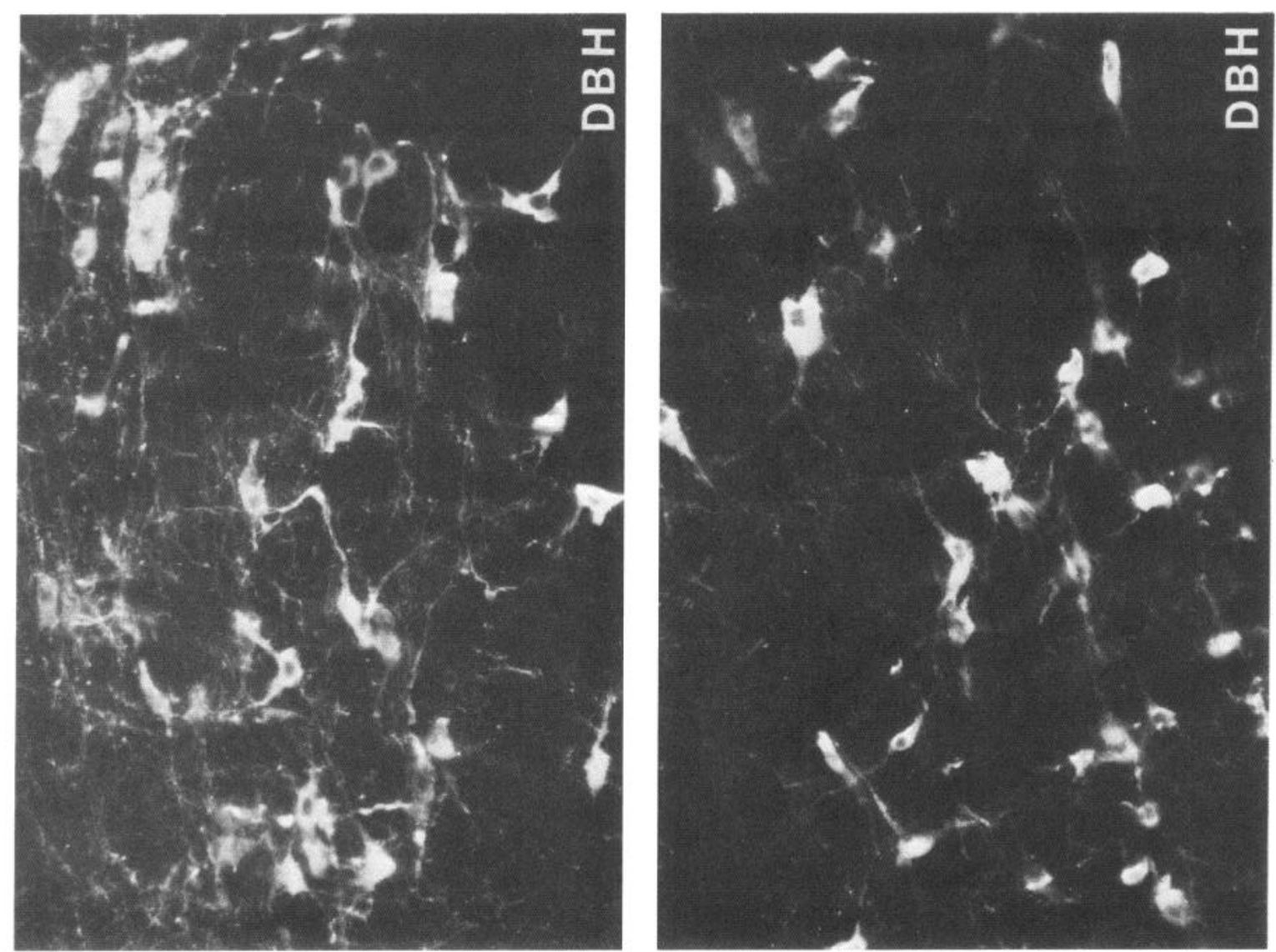

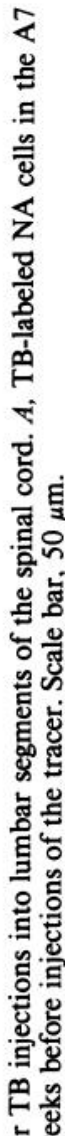
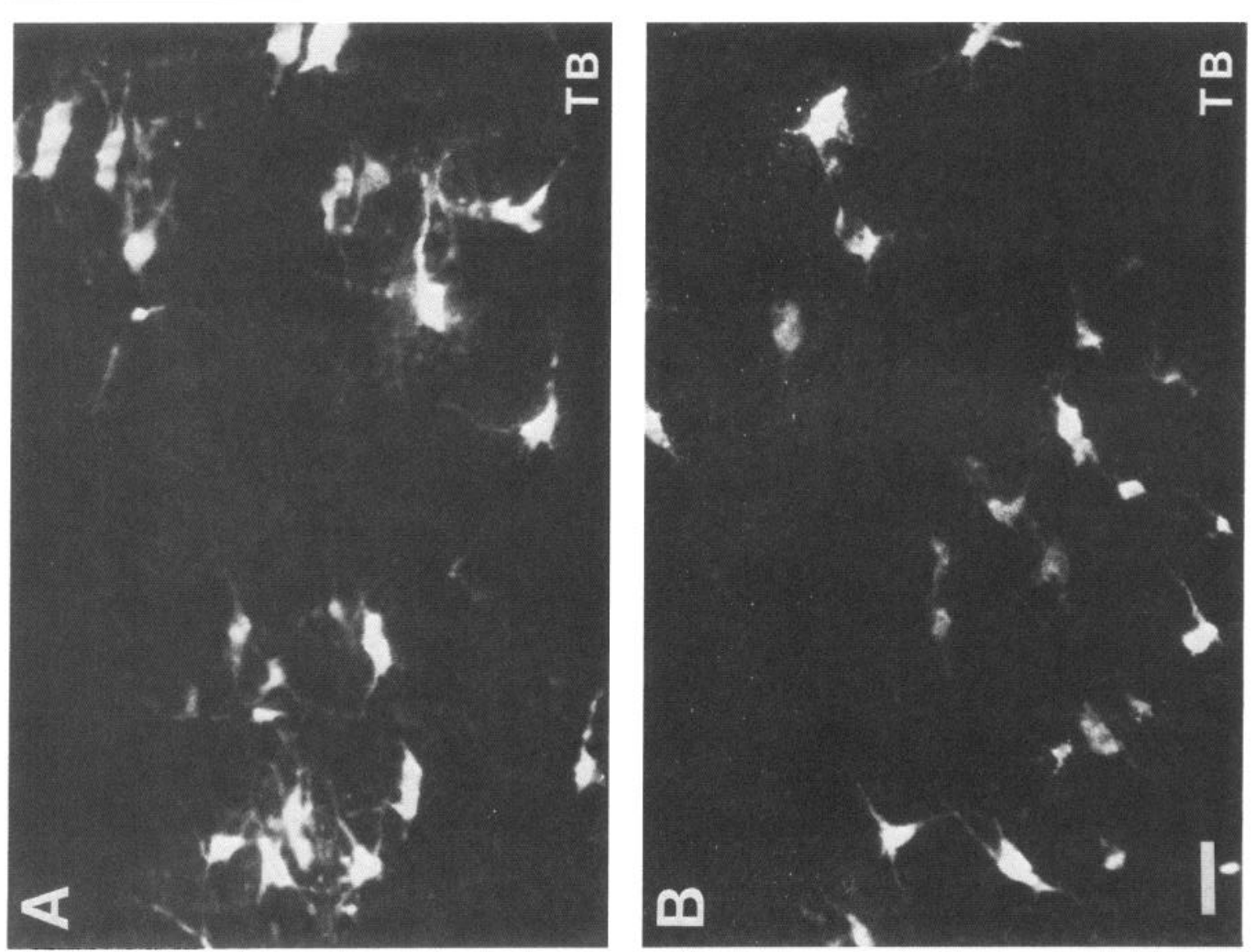

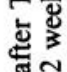

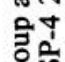

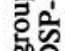

近

空

$\Xi$ 退



完

害号

땡음

눙

它尊

도.

ํํㅇ

递

हू

응

응

ฮั

乐

远

항

형

$\forall$

$+\div$

홍응 
summarizes the results of the retrograde labeling of NA cells in control and drug-treated rats.

\section{Discussion}

Previous biochemical studies of the neurotoxic effects of DSP-4 on NA axons described a preferential action of the drug on axons originating in cells of the LC (Jonsson et al., 1981; Jonsson, 1983; Hallman et al., 1984). In an immunohistochemical analysis of the effects of DSP-4 on NA axon staining in the rat CNS (Fritschy and Grzanna, 1989), we demonstrated a profound selectivity of DSP-4 for NA axons of the LC. Administered at doses that produce complete degeneration of LC axons, DSP-4 has little or no effect on the structural integrity of NA axons of non-LC origin. In this study we have taken advantage of this differential response of NA axons to DSP-4 to identify the origin of NA axon terminals in the dorsal and ventral horn of the spinal cord.

\section{$N A$ axons in spinal cord that remain after systemic DSP-4 treatment}

The histochemical analysis of the effects of DSP-4 treatment on NA axon staining in the spinal cord confirms our earlier observation that the drug produces a nearly complete loss of NA axon staining in the dorsal horn and intermediate zone at all levels of the spinal cord. In contrast, DSP-4 treatment results in an only slight reduction in NA axon staining in the ventral horn. These spared NA fibers are found in the neuropil of the motoneuron layer, lamina IX (Paxinos and Watson, 1986), and the intermediolateral cell column (Figs. $1 B, 2 B$ ). We did not observe any noticeable change in the staining of NA axons in the ventral horn between 2 and 4 weeks after DSP- 4 treatment. However, preliminary observations in studies of effects of DSP-4 3 months after drug treatment revealed further loss of NA axon terminals in lamina IX. If this observation can be confirmed, it would suggest that NA axons innervating somatic motoneurons degenerate at a much slower rate than NA axons in the dorsal horn and the intermediate zone.

A differential sensitivity of descending NA axons to a neurotoxin has previously been observed by McBride et al. (1985), who found that neonatal 6-hydroxydopamine destroys spinal cord noradrenergic axons from the LC but not those from the lateral tegmental cell groups. These authors observed an $85 \%$ decrease in the number of retrogradely labeled LC neurons after tracer injections into thoracic segments of the spinal cord of rats treated with 6-hydroxydopamine at birth. This result agrees well with our finding of a more than $90 \%$ loss of labeling after DSP4. In their study, McBride et al. (1985) did not find any decrease in the number of retrogradely labeled neurons in other NA subgroups of the pons. However, these investigators identificd A5 and A 7 cells based upon their location in the lateral pontine tegmentum rather than by immunohistochemical staining. We have consistently found a number of non-NA cells among A7 cells with projections to the spinal cord, and it is possible that McBride et al. (1985) may have overestimated in their study the number of retrogradely labeled NA cells in the A5 and A7 groups.

There is a conspicuous difference in the morphology of NA axon terminals ablated by DSP-4 and those spared by the drug. While NA axons of the dorsal horn and the intermediate zone (Figs. $1 A$ and $2 A$ ) have distinct intervaricose segments, NA axon terminals in lamina IX (Figs. $1 B$ and $2 B$ ) typically have a dotlike appearance. Thus, 2 populations of NA axons can be distin-
Table 1. Retrograde labeling of NA cells in DSP-4-treated rats after TB injections into thoracic (mean of 4 cases) and lumbar segments (mean of 2 cases) of the spinal cord.

\begin{tabular}{lll}
$\begin{array}{l}\text { Spinal cord } \\
\text { level }\end{array}$ & NA subgroup & $\%$ \\
\hline Thoracic & A5 caudal & Control \\
\hline & A5 rostral & 52 \\
& A7 & 32 \\
& subcoerulus & 51 \\
Lumbar & locus coeruleus & 39 \\
& A5 caudal & 7 \\
& A5 rostral & 65 \\
& A7 & 57 \\
& subcoeruleus & 63 \\
& locus coeruleus & 47 \\
\hline
\end{tabular}

guished in the spinal cord based upon their differential sensitivity to DSP-4 and based upon their morphologic features. Two groups of NA axons have previously been identified by Levitt and Moore (1979). Their fluorescence histochemical analysis led them to conclude that in the brain stem $L C$ axons are readily distinguished from non-LC NA axons based upon the size, shape, and spacing of their varicosities.

\section{Topography of descending NA projections}

The LC, subcoeruleus, and the A5 and A7 groups have previously been identified as the source of the NA projections to the spinal cord (Westlund and Coulter, 1980; Stevens et al., 1982; Westlund et al., 1983; Byrum et al., 1984; Lyons and Grzanna, 1988). The existence of a topographic order in the descending NA projections to the monkey spinal cord has been proposed by Westlund and Coulter (1980). The results of this study reveal a definite topographic order in the descending projections from the LC and from NA cells of the A5 and A7 groups in the rat. The loss of NA axon staining in the dorsal horn and the intermediate zone after DSP-4 treatment is accompanied by a nearly complete loss of retrograde labeling of LC cells (Fig. 3) but only a moderate reduction in the labeling of $\mathrm{A} 5$ and $\mathrm{A} 7$ cells. We interpret this finding as evidence that spinal cord regions devoid of NA axon staining after DSP-4 treatment are regions supplied by LC axons. The retrograde labeling of $A 5$ and $A 7$ cells must be attributed entirely to transport of TB by the remaining NA axons in the ventral horn and the intermediolateral cell column. Based on the results of this study, we cannot rule out that A5 and $A 7$ cells also project to the dorsal horn and the intermediate zone of the spinal cord. However, if this were the case, it would make it necessary to postulate the existence of A5 and A7 cell axons in the spinal cord with different susceptibilites to DSP-4.

We have been unable to determine whether the NA projections to the ventral horn of lumbar spinal cord and the intermediolateral cell column of the thoracic spinal cord originate in separate NA subgroups. That the NA innervation of the intermediolateral cell column originates primarily, if not exclusively, in cells of the A5 group has previously been suggested (Byrum et al., 1984; I oewy et al., 1986). We have proposed recently (Lyons and Grzanna, 1988) that cells of the A7 group may be the principal source of the NA innervation of motoneurons. In the present study, we did not observe any significant difference in the labeling of A 5 and A 7 cells after tracer injections 
into thoracic and lumbar levels of the spinal cord. This finding may reflect the fact that both NA subgroups project to the ventral horn and the intermediolateral cell column of the thoracic spinal cord. However, the data would also be compatible with the interpretation that $A 5$ cells project mainly to visceromotor neurons while A7 cells project to somatic motoneurons, since our lumbar injections would have labeled descending A5 axons on their way to sacral spinal cord levels. The origin of the NA innervation of the parasympathetic neurons at sacral levels is still controversial (Loewy et al., 1979; Westlund and Coulter, 1980; Hida and Shimizu, 1982), and further studies will be needed to determine whether the NA innervation of the preganglionic sympathetic and parasympathetic neurons originate from the same or different NA subgroups.

Our conclusion that cells of the LC project to the dorsal horn and the intermediate zone, while NA cells of the A5 and A7 groups project to somatic motoneurons and preganglionic neurons of the spinal cord contradicts the findings of several earlier studies. Based upon biochemical and histochemical studies, it has been concluded that the LC distributes axons to the ventral horn, the intermediate zone, and the ventral part of the dorsal horn (Nygren and Olson, 1977; Commissiong et al., 1978; Commissiong, 1981). Anterograde transport studies also concluded that the coeruleospinal projections (Kuypers, 1982; Holstege and Kuypers, 1987) terminate widely throughout all parts of the spinal cord gray matter but most heavily among motoneuronal cell groups. Projections of the LC to both the superficial laminae of the dorsal horn as well as to the ventral horn have been described in the rat (Hida and Shimizu, 1982), the opossum (Martin et al., 1979a), and the monkey (Westlund and Coulter, 1980). While we cannot discount the possibility, based upon the present data alone, that the LC distributes a small contingent of axons to the ventral horn, this arrangement seems unlikely. Support for this conclusion is provided by the results of an anterograde transport study in which we used the lectin PHA-L in combination with DBH immunohistochemistry to determine the distribution of LC axons in the rat spinal cord (Fritschy et al., 1987). In that study we provided direct evidence that the LC distributes axons throughout the dorsal horn and the intermediate zone; in contrast, few LC axons could be traced into the ventral horn and none into the vicinity of motoneuron pools. The region found to be devoid of I.C axons in the study by Fritschy et al. (1987; see their figure 4) precisely coincides with the terminal plexus of NA axons spared by DSP-4 treatment in this study. Thus, the data of the 2 studies complement each other and together provide a substantial body of evidence for the proposition that the LC innervates the dorsal horn and intermediate zone of the spinal cord and that NA cells of the $A 5$ and $A 7$ groups project to the ventral horn and the intermediolateral cell column.

Based upon studies of the effects of bilateral LC lesions on the distribution of NA axons in the brain stem, Levitt and Moore (1979) concluded that the LC innervates sensory and association areas, whereas non-LC cells innervate motor nuclei at brainstem levels. We have shown by retrograde transport (Grzanna et al., 1987) that the motor nuclei of the trigeminal and facial nerve receive their NA inputs almost exclusively from cells of the A5 and A7 groups, with little or no contribution from the LC. We have also shown in a double-retrograde transport study that the same A5 and A 7 cells innervating the motor trigeminal nucleus also project to the spinal cord (Lyons and Grzanna, 1988). Thus, NA neurons in the ventrolateral pons may be the only NA cells that can influence directly somatic motor and visceral motor neurons.

Since the identification of norepinephrine in the spinal cord (Euler, 1946) and the discovery of its supraspinal origin (Magnussen and Rosengren, 1963; Carlsson et al., 1964), this aminergic bulbospinal system has been suspected to influence a wide range of spinal cord functions (for review, see Bjorklund and Skagerberg, 1982; Kuypers, 1982; Bjorklund and Lind vall, 1986). This study provides direct evidence that the bulbospinal NA system consists of at least 2 separate and distinct descending pathways. These 2 pathways differ in their origin and termination within the spinal cord and should therefore be considered as 2 separate anatomical systems with entirely different functional capacities.

\section{References}

Berod, A., B. K. Hartman, and J. F. Pujol (1981) Importance of fixation in immunohistochemistry: Use of formaldehyde solutions at variable $\mathrm{pH}$ for the localization of tyrosine hydroxylase. J. Histochem. Cytochem. 29: 844-850.

Bjorklund, A., and O. Lindvall (1986) Catecholaminergic brain stem regulatory systems. In Handbook of Physiology, Sect. 1, Vol. 4, V. B. Mountcastle, sect. ed., F. E. Bloom, vol. ed., pp. 155-235, American Physiological Society, Bethesda, MD.

Bjorklund, A., and G. Skagerberg (1982) Descending monoaminergic projections to the spinal cord. In Brain Stem Control of Spinal Mechanisms, B. Sjolund and A. Bjorklund, eds., pp. 55-88, Elsevier, New York.

Blessing, W. W., A. K. Goodchild, R. A. L. D. Dampney, and J. P. Chalmers (1981) Cell groups in the lower brain stem of the rabbit projecting to the spinal cord with special reference to catecholaminecontaining neurons. Brain Res. 221: 35-55.

Byrum, C. E., R. Stornetta, and P. G. Guyenet (1984) Electrophysiological properties of spinally-projecting A5 noradrenergic neurons. Brain Res. 303: 15-29.

Carlsson, A., B. Falck, K. Fuxe, and N.-A. Hillarp (1964) Cellular localization of monoamines in the spinal cord. Acta Physiol. Scand. 60: $112-119$.

Commissiong, J. W. (1981) Evidence that the noradrenergic coeruleospinal projection decussates at the spinal level. Brain Res. 212: 145-151.

Commissiong, J. W., S. O. Hellstrom, and N. H. Neff (1978) A new projection from the locus coeruleus to the spinal ventral columns: Histochemical and biochemical evidence. Brain Res. 148: 207-213.

Dahlstrom, A., and K. Fuxe (1964) Evidence for the existence of monoamine-containing neurons in the central nervous system. I. Demonstration of monoamines in the cell bodies of brain stem neurons. Acla Pliysiol. Scand. (Suppl. 62) 232: 1-55.

Dahlstrom, A., and K. Fuxe (1965) Evidence for the existence of monoamine neurons in the central nervous system. II. Experimentally induced changes in the intraneuronal amine levels of bulbospinal neuron systems. Acta Physiol. Scand. (Suppl. 64) 247: 1-36.

Euler, U.S. (1946) A specific sympathomimetic ergone in adrenergic nerve fibers (sympathin) and its relationship to adrenaline and noradrenaline. Acta Physiol. Scand. 12: 73-97.

Fritschy, J.-M., and R. Grzanna (1989) Immunohistochemical analysis of the neurotoxic effects of DSP-4 identifies two populations of noradrenergic axon terminals. Neuroscience (in press).

Fritschy, J.-M., W. E. Lyons, C. A. Mullen, B. E. Kosofsky, M. E. Molliver, and R. Grzanna (1987) Distribution of locus coeruleus axons in the rat spinal cord: A combined anterograde transport and immunohistochemical study. Brain Res. 437: 176-180.

Gerfen, C. R. (1985) The neostriatal mosaic. I. Compartmental organization of projections from the striatum to the substantia nigra in the rat. J. Comp. Neurol. 236: 454-476.

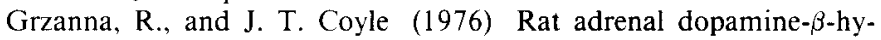
droxylase: Purification and immunologic characteristics. J. Neurochem. 27: 1091-1096.

Grzanna, R., W. K. Chee, and E. W. Akeyson (1987) Noradrenergic projections to brainstem nuclei: Evidence for differential projections from noradrenergic subgroups. J. Comp. Neurol. 263: 76-91.

Hallman, H., E. Sundstrom, and G. Jonsson (1984) Effects of the 
noradrenalıne neurotoxin DSP4 on monoamine neurons and their transmitter turnover in rat CNS. J. Neural Transm. 60:89-102.

Hida. T.. and N. Shimizu (1982) The interrelation between the laterodorsal tegmental area and lumbosacral segments of rats as studied by HRP method. Arch. Histol. Jpn. 45: 495-504.

Holstege. J. C., and H. G. J. M. Kuypers (1987) Brainstem projections to spinal motoneurons: An update. Neuroscience 23: 809-821.

Holstege. (i., H. G. J. M. Kuypers, and R. C. Boer (1979) Anatomical evidence for direct brain stem projections to the somatic motoneuronal cell groups and autonomic preganglionic cell groups in cat spinal cord. Brain Res. 171: 329-333.

Hsu. S. M., L. Raine, and H. Fanger (1981) Use of avidin-biotinperoxidase complex (ABC) in immunoperoxidase techniques: A comparison between $\mathrm{ABC}$ and unlabeled antibody (PAP) procedures. J. Histochem. ('ytochem. 29: 577-580.

Jonsson. G. (1983) Chemical lesioning techniques: Monoamine neurotoxins. In Handbook of Chemical Neuroanatomy, A. Bjorklund and T. Hokfelt. eds., pp. 463-507, Elsevier. Amsterdam.

Jonsson. G.. H. Hallman, F. Ponzio, and S. Ross (1981) DSP4 (N-(2chloroethyl)-N-ethyl-2-bromobenzylamine)-A useful denervation tool for central and peripheral noradrenalin neurons. Eur. J. Pharmacol. 72: 173-188.

Kuypers. H. G. J. M. (1982) Anatomy of descending pathways to the spinal cord. Prog. Brain Res. 57: 371-403.

Levitt. P.. and R. Y. Moore (1979) Origin and organization of brainstem catecholamine innervation in the rat. J. Comp. Neurol. 186: 505-528.

Loewy, A. D.. C. B. Saper, and R. P. Baker (1979) Descending projections from the pontine micturition center. Brain Res. 172: $533-$ 538.

Loewy. A. D., L. Marson, D. Parkinson, M. A. Perry, and W. B. Saywer (1986) Descending noradrenergic pathways involved in the A5 depressor response. Brain Res. 386: 313-324.

Lyons. W. E.. and R. Grzanna (1988) Noradrenergic neurons with divergent projections to the motor trigeminal nucleus and the spinal cord: A double retrograde neuronal labeling study. Neuroscience 26 . 681-693.

Magnusson. T., and E. Rosengren (1963) Catecholamines of the spinal cord normally and after transection. Experientia 14: 229-230.

Martin. G. F.. A. O. I Iumbertson. Jr., L. C. Laxson, and W. M. Panneton (1979a) Dorsolateral pontospinal systems. Possible routes for catecholamine modulation of nociception. Brain Res. 163: 333-338.
Martin, G. F., A. O. Humbertson, Jr., L. C. Laxson, W. M. Panneton, and I. Tschismadia (1979b) Spinal projections from the mesencephalic and pontine reticular formation in the North American opossum: A study using axonal transport techniques. J. Comp. Neurol. 194: $373-400$

McBride. R. L., R. V. Ozment. and J. Sutin (1985) Neonatal 6-hydroxydopamine destroys spinal cord noradrenergic axons from the locus coeruleus, but not those from lateral tegmental cell groups. J. Comp. Neurol. 235: 375-383.

Mizukawa, K. (1980) The segmental detailed topographical distribution of monoaminergic terminals and their pathways in the spinal cord of the cat. Anat. Anz. 147: 125-144.

Nygren, L.-G., and L. Olson (1977) A new major projection from the locus coeruleus: The main source of noradrenergic nerve terminals in the ventral and dorsal columns of the spinal cord. Brain Res. 132: 85-93.

Paxinos, G., and C. Watson (1986) The Rat Brain in Stereotaric Coordinates, Academic, New York

Ross, S. B., J. G. Johansson, B. Lindborg, and R. Dahlbom (1973) Cyclizing compounds. I. Tertiary N-(2-bromobenzyl)-N-haloalkylamines with adrenergic blocking action. Acta Pharm. Suecica 10:2942.

Satoh, K., M. Tohyama, K. Yamamoto, T. Sakumoto, and N. Shimizu (1977) Noradrenaline innervation of the spinal cord studied by the horseradish peroxidase method combined with monoamine oxidase staining. Exp. Brain Res. 30: 175-186.

Stevens, R. T., C. H. Hodge, Jr., and A. V. Apkarian (1982) KollikerFuse nucleus: The principal source of pontine catecholaminergic cells projecting to the lumbar spinal cord of cat. Brain Res. 239: 589-594.

Westlund, K. N., and J. D. Coulter (1980) Descending projections of the locus coeruleus and subcoeruleus/medial parabrachial nuclei in monkey: Axonal transport studies and dopamine- $\beta$-hydroxylase immunocytochemistry. Brain Res. Rev, 2; 235-264.

Westlund, K. N., R. M. Bowker, M. G. Ziegler, and J. D. Coulter (1983) Noradrenergic projections to the spinal cord of the rat. Brain Res. 263: 15-31.

Zieher, L. M. and G. Jaim-Etcheverry (1980) Neurotoxicity of N-(2chloroethyl)-N-ethyl-2-bromobenzylamine hydrochloride (DSP 4) on noradrenergic neurons is mimicked by its cyclic aziridinium derivative. Eur. J. Pharmacol. 65: 249-256. 